\title{
Editorial
}

\section{Surgical management of the neonate with congenital heart disease}

\author{
MARC R DE LEVAL \\ From the Thoracic Unit, The Hospital for Sick Children, Great Ormond Street, London
}

Despite the multiplication of specialised centres and a fall in the birth rate, paediatric cardiac units have seen a steady increase in the number of infants with congenital heart defects operated on during the neonatal period. This changing pattern is the result of continuing advances in neonatology, early detection and better management of the neonate with a congenital heart defect, and the tendency, rightly or wrongly, to operate on more complex defects.

Advances in neonatology with improved infant care and ventilatory support for infants with respiratory distress syndrome have increased the neonatal survival rate. Hence the increase in the number of premature infants with a persistent ductus ateriosus who require surgical closure, often after a trial of treatment with indomethacin.

More specifically the preoperative status of neonates with congenital heart defects has been greatly improved by the introduction of prostaglandins and a trend to non-invasive diagnosis. Conventional clinical diagnosis of congenital heart defects by physical examination, chest radiography, and electrocardiography is often unreliable, particularly in the neonatal period. For this reason cardiac catheterisation and angiocardiography have for many years been the standard diagnostic methods in this age group. Cross sectional echocardiography has changed the indications and need for cardiac catheterisation. Neonates have a technically excellent subcostal and suprasternal window that allows good definition of the intracardiac anatomy. Most congenital cardiac malformations can be diagnosed by cross sectional echocardiography in the neonate. The anatomical definition of the anomaly together with

Requests for reprints to Dr Marc R de Leval, Thoracic Unit, The Hospital for Sick Children, Great Ormond Street, London WCIN 3JH. the physical findings are often sufficient to proceed to surgical treatment in the absence of haemodynamic information from a cardiac catheterisation. We have operated on numerous neonates with various heart defects when all non-invasive investigations were typical of the suspected abnormality. The type of surgery to which the patient is to be submitted must also be considered in this decision. For example, the preoperative information required for a shunt procedure differs from that required for a complete repair. Is the risk of preoperative invasive investigation of a sick neonate justified by its greater accuracy compared with alternative non-invasive investigations? This question cannot be answered unless risk and accuracy can be expressed in the same units, for only then can they be usefully compared.

Pharmacological manipulation of the ductus arteriosus with prostaglandins has been a breakthrough in the management of cyanotic neonates with reduced pulmonary blood flow. Operations are now performed more electively and infants reach the operating theatre in a better condition, that is warm, well oxygenated, non-acidotic, and with a good cardiac output. Prostaglandins have been particularly beneficial for patients with pulmonary atresia with or without ventricular septal defect, for those with tricuspid atresia, and to some extent for those with transposition of the great arteries. They have also been used for aortic arch anomalies, coarctation of the aorta, or critical aortic valve stenosis in which part of the systemic circulation may depend on the patency of the ductus arteriosus. The exact mechanism by which prostaglandins afford haemodynamic improvement in patients with obstructive lesions of the systemic circulation is not completely understood except in conditions in which the systemic circulation is entirely duct dependent.

Refinements of well established surgical pro- 
cedures and their implementation as logical management protocols have also contributed to changes in neonatal cardiac surgery. The management of neonatal coarctation of the aorta and critical aortic stenosis may be taken as examples.

The acutely ill neonate with coarctation of the aorta requires urgent treatment. The prognosis is especially poor when the coarctation is associated with intracardiac anomalies, of which ventricular septal defect is the most common. In general these patients tend to have intractable heart failure, metabolic acidosis, and renal failure. Formerly we considered these two cardiac anomalies to be entities and we treated each one separately. The coarctation was repaired first and then, if the patient remained in intractable heart failure, the ventricular septal defect was repaired. Now we know that neonates with a large ventricular septal defect are more likely to survive if banding of the pulmonary artery is carried out as well as repair of the coarctation. Though it is not a panacea against late complications, the subclavian flap angioplasty technique to relieve coarctation of the aorta has been a useful additional surgical treatment.

Aortic valvar stenosis that is severe enough to produce left ventricular failure in the neonatal period is associated with a high mortality rate. Medical treatment is only briefly effective and surgical intervention is mandatory for survival. During the past five years we have evolved a protocol for these infants which includes a non-invasive diagnosis, followed by valvotomy without cardiopulmonary bypass. This is done either under inflow occlusion through a transaortic approach, or by transapical dilatation of the valve. We have a very limited experience with balloon dilatation of the aortic valve, but this procedure might become the method of choice for critical aortic stenosis in the neonate.

Open heart surgery during the neonatal period has made little advance during the past decade. Studies of biochemical and immunological reactions induced by extracorporeal circulation and a better understanding of the function and protection of the neonatal myocardium should lead to safer cardiac surgery. Repair of a total anomalous pulmonary venous connection is one of the commonest open heart procedures performed in the neonate. After the sharp reduction in the surgical mortality that followed the revival of deep hypothermia and circulatory arrest in the early 1970 s the mortality for this condition remains greater than $30 \%$. Patients under one week of age with obstruction of pulmonary venous return have a particularly poor prognosis. Intrinsic anomalies of the pulmonary veins and of the lung parenchyma may account for these disappointing results.
New trends in the management of complete transposition of the great arteries illustrate the impact made by innovative surgery. Though intra-atrial repair of transposition of the great arteries by either the Mustard or Senning techniques has been widely used for many years, there is concern about the very long term results of these interventions. Jatene and colleagues introduced the arterial switch or so called anatomical repair of transposition of the great arteries. ${ }^{1}$ This was successfully applied to transposition with raised left ventricular pressure due to either a large left to right shunt or dynamic left ventricular outflow tract obstruction. The concept was more recently extended to the correction of simple transposition of the great arteries during the neonatal period at a time when the pulmonary vascular resistance is still so high that the left ventricle is capable of sustaining the systemic circulation after the switch procedure. The early results of this management have been very encouraging. The prospect of improved long term results would justify a wider use of this approach if late complications could be avoided. Because this remains a hypothetical prospect some units, such as ours, continue to be rather conservative about the management of simple transposition of the great arteries.

Progress in neonatology, the availability of reliable pharmacological and surgical means to regulate the pulmonary blood flow, and the fundamental demonstration that the pulmonary and the systemic circulations left in series could be successfully served by one ventricle (Fontan operation) have made the most complex cardiac malformations amenable to surgery. These developments account for changes in the management of hypoplastic right and left heart syndromes. The latter is the fourth most common congenital cardiac anomaly seen in infancy (after ventricular septal defect, tetralogy of Fallot, and transposition of the great arteries) and is responsible for nearly $25 \%$ of cardiac deaths during the first week of life. Closure of the arterial duct in the first few days of life explains the early death of so many neonates with hypoplastic left heart syndromes. On the assumption that hypoplastic left heart syndrome is very similar to extreme forms of right ventricular hypoplasia, tricuspid atresia, and other forms of univentricular hearts Norwood and his colleagues have developed palliative procedures for such patients. ${ }^{2}$ The purpose of these interventions is to establish a permanent communication between the right ventricle and the aorta, regulate the pulmonary blood flow (avoiding pulmonary vascular changes secondary to increased flow and pressure), and to assure a satisfactory interatrial communication. The definitive repair consists of separating the systemic and pulmonary circulations by performing a Fontan type 
of procedure. We have followed with interest the evolution and the careful and systematic study of Norwood and his colleagues. ${ }^{2}$ We believe that such a planned research programme should be supported but we think that it is premature for all surgical teams to embark on these often heroic surgical treatments without their knowing how many of the survivors of the first stage operation will eventually be suitable for a Fontan procedure. If their numbers are very small is it ethically justifiable to oblige patients who would otherwise have died as neonates to carry their handicap into childhood only to succumb because no further treatment is possible?

Neonatal cardiac transplantation with a homograft or even a xenograft heart is an alternative approach to treatment of patients with these complex malformations. Both procedures have already been performed. Are these pioneering endeavours going to open the doors to new hopes or create formidable problems?

One can predict that in the foreseeable future a number of researches that are currently underway will deeply influence the incidence and the management of neonates with congenital heart defects. Some workers are investigating the possibilities of operating on fetal hearts in utero because they consider that the womb is likely to be a better postoperative environment than the most advanced incubators. In several centres intrauterine echocardiographic diagnosis of complex cardiac malformations has led to the termination of pregnancy. In future the attitude towards the treatment of complex cardiac malformations in neonates will be based on the quality of adult life enjoyed by patients operated on today and on the outcomes of these two conflicting and radical tendencies, that is to replace the defective organ or to prevent the birth of the faulty embryo. Ethical, social, and economic considerations must also influence this subtle decision making.

\section{References}

1 Jatene AD, Fontes VF, Paulista PP, et al. Anatomic correction of transposition of the great arteries. f Thorac Cardiovasc Surg 1976; 72: 364-70.

2 Norwood WI, Lang P, Castaneda AR, Campbell DN. Experience with operations for hypoplastic left heart syndrome. F Thorac Cardiovasc Surg 1981; 82: 511-9. 